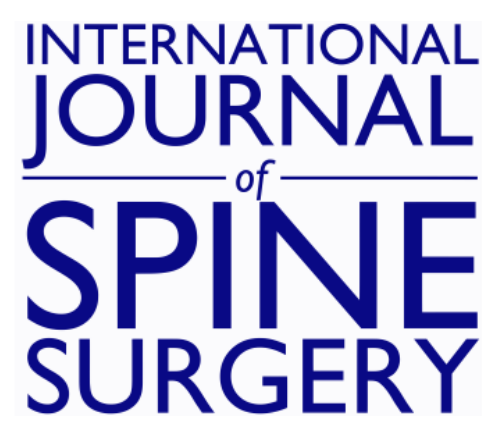

\title{
Electromagnetic Navigation in Minimally Invasive Spine Surgery: Results of a Cadaveric Study to Evaluate Percutaneous Pedicle Screw Insertion
}

Justin F. Fraser, Ron Von Jako, John A. Carrino and Roger Härtl

Int J Spine Surg 2008, 2 (1) 43-47

doi: https://doi.org/10.1016/SASJ-2007-0105-RR

http://ijssurgery.com/content/2/1/43

This information is current as of April 26, 2023.

Email Alerts Receive free email-alerts when new articles cite this article. Sign up at: http://ijssurgery.com/alerts 


\title{
Electromagnetic Navigation in Minimally Invasive Spine Surgery: Results of a Cadaveric Study to Evaluate Percutaneous Pedicle Screw Insertion
}

Justin F. Fraser, $M D$, Ron Von Jako, MD, John A. Carrino, MD, MPH, and Roger Härtl, MD

\begin{abstract}
Background

This cadaveric study compared efficacy and safety of an electromagnetic (EM) guidance system versus conventional fluoroscopy for percutaneous pedicle screw fixation. As percutaneous pedicle screw fixation becomes increasingly common in spinal surgery, intraoperative imaging systems that maximize efficiency while minimizing radiation exposure and inaccurate trajectories will be progressively more important. Published studies have validated the safety of percutaneous screw fixation using conventional fluoroscopic guidance and frameless optical stereotaxy, though EM guidance systems have not been evaluated for percutaneous placement in the lumbosacral spine. The aim of the study was to evaluate the clinical applicability of an EM system for minimally invasive spine fusion in the lumbosacral spine.
\end{abstract}

\section{Methods}

Five human cadaveric specimens underwent bilateral lumbosacral percutaneous screw fixation from L1 to S1 using conventional anteroposterior (AP) and lateral fluoroscopic techniques on one side and 2-dimesional (2D) EM guidance on each matching side. Intraoperative efficiency was evaluated, and pedicle, vertebral, and critical breach rates were assessed on postoperative computed tomography (CT).

\section{Results}

Overall mean fluoroscopy time per screw was $58.9 \pm 44.7$ seconds for conventional fluoroscopy compared to $27.4 \pm 13.5$ seconds for electromagnetic guidance $(P=.0003)$. Pedicle, vertebral, and critical breach rates for the L1-S1 were $32.1 \%, 10.7 \%$, and $25.0 \%$ for conventional fluoroscopy and $42.8 \%, 10.7 \%$, and $14.1 \%$ for electromagnetic guidance (difference not statistically significant [ns]). In comparing critical breaches in the lumbar spine (L1-L5), there was a significant difference between 2-D EM guidance (0) and CF guidance (6) $(P=.02)$.

\section{Conclusions}

Two-dimensional EM navigation provides a modality for lumbosacral percutaneous pedicle screw fixation that is more efficient and safer than conventional fluoroscopy. This data provides a foundation for further clinical trials of this technology.

\section{Level of Evidence}

Level 5 - Bench Research

Key Words: Minimally invasive spine fusion, percutaneous pedicle screw fixation, electromagnetic guidance. SAS Journal. Winter 2008;2:43-47. DOI: SASJ-2007-0105-RR

\section{INTRODUCTION}

Recent advances in minimally invasive spine surgery have spawned new interest in utilizing percutaneous device placement to accomplish spinal decompression and fixation that previously could only be achieved through large, open incisions. As this area of spine surgery develops, novel tools are needed to maximize efficiency and accuracy. Without open exposure, image-guided surgery (IGS) becomes increasingly important for safe and effective spine surgery. The spine surgeon becomes dependent upon visualization of spinal anatomy through indirect methodologies such as fluoroscopy to provide a virtual and real-time anatomical map to chart the course of placed instrumentation.
Recently published research has focused on methodologies for ensuring precise and efficient percutaneous pedicle screw fixation. Wiesner et al. demonstrated a pedicle screw misplacement rate of $6.6 \%$ among 408 percutaneously placed screws in 54 patients. ${ }^{1}$ Of the 27 misplaced screws, only one screw-related nerve root injury was reported. Laine et al., in a study comparing conventional pedicle screw fixation using anatomic landmarks in 50 patients (277 screws) to computerassisted screw fixation using an optoelectronic navigation system in 41 patients ( 219 screws), found a significant difference in pedicle perforation rate (13.4\% conventional versus $4.6 \%$ computer-assisted, $P=.006){ }^{2}$ Several other groups have utilized optical tracking systems that use dynamic reference 
frames to provide real-time 3-dimensional guidance in pedicle screw placement. ${ }^{3,4}$ More recently, Sagi et al., in a cadaveric study comparing electromagnetic field-based (EM) image guidance to conventional anatomical/fluoroscopic guidance, found similar rates of screw misplacement in the thoracic spine. ${ }^{5}$ EM tracking, as an alternative to optical tracking, significantly reduced mean screw insertion time per screw (179 versus 261 seconds, $P=.007$ ) and mean total fluoroscopy time (162 versus 261 seconds, $P=.045) .{ }^{5}$ Thus, EM tracking technology, which, unlike optical imaging, does not depend on continuous line-ofsight registration, represents an important development in the advance of image-guided complex spine surgery. While Sagi et al. demonstrated the utility of EM guidance in open pedicle screw placement in the thoracic and lumbar spine, its utilization for lumbosacral percutaneous screw placement has yet to be tested. ${ }^{5,6}$ Therefore, the goal of our cadaveric study was to evaluate surgical precision and efficiency of an electromagnetic field image guidance system in percutaneous lumbar pedicle screw insertion compared to conventional fluoroscopic image guidance.

\section{MATERIALS AND METHODS}

This study was a human cadaveric anatomical laboratory investigation; 5 human cadaveric specimens were included. Two imaging systems were used and compared: (1) the GE NAV Gold Sensor system (GE Healthcare, InterventionalOEC, Salt Lake City, Utah) with an IT3500 platform and (2) a conventional biplanar fluoroscopy using an OEC9800 image intensifier (GE Healthcare, Interventional-OEC). The specimens were prescreened to eliminate significant deformity such as fracture, scoliosis, or spondylolisthesis. The specimens were each placed on a Jackson table in the prone position for posterior access. Each cadaver was subject to screw placements from L1 to S1 (inclusive); K-wires were applied bilaterally at each level. For comparative analysis, screws were placed on one side of each cadaver utilizing conventional fluoroscopic technique alone, and an equal number of screws were placed on the opposite side by the same surgeon using the EM-based navigational system.

For the conventional fluoroscopy group (CF), K-wires were inserted via a Jamshidi needle using multiple anteroposterior (AP) and lateral views to localize the pedicle at each level. For the EM group (EM), K-wires were inserted using the EM navigation system, pictured in Figure 1A. An EM transmitter was attached rigidly to a spinous process (Figure 1B), producing 3 orthogonal EM fields encompassing the anatomical field (Figure 1C). AP and lateral views of each target spinal segment were obtained using the image intensifier outfitted with a receiveroutfitted calibration grid that interacts with the EM surgical field. Surgical instruments were custom-made and precalibrated to the EM transmitter. With calibration, instruments could be tracked in real-time AP, lateral, and oblique image displays as illustrated in Figure 1B. Using this tracking method on the EMside and "free-hand" fluoroscopic placement on the control side, a navigated precalibrated GE NAV Access Needle (Jamshidi

\section{Figure 1. EM Navigation System}

A

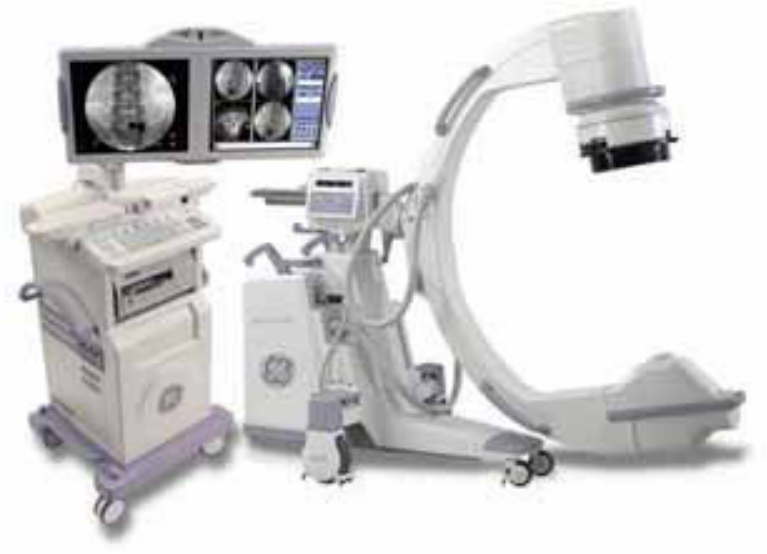

B

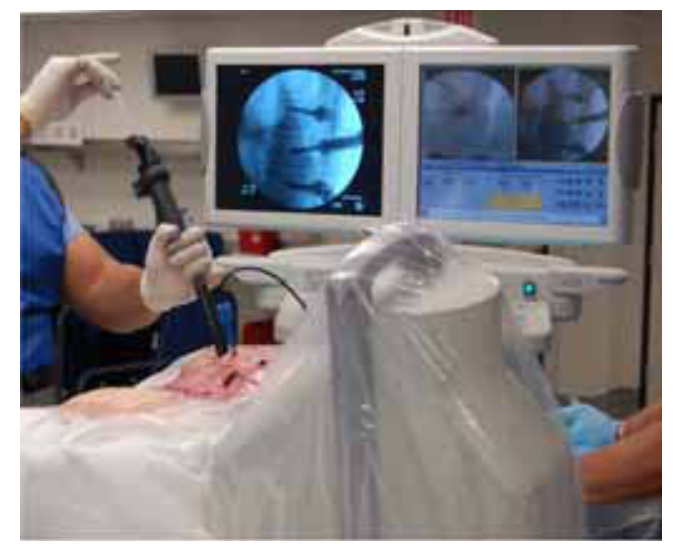

C

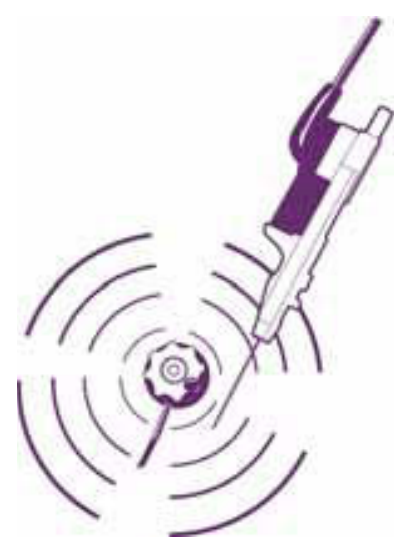

The EM navigation system is the size and profile of a standard fluoroscopy C-arm, but has stereotactic capability (A). The cadaveric demonstration of percutaneous lumbar pedicle screw placement illustrates the EM transmitter and a precalibrated instrument tracking on the 2D field-of-view (B). An illustration of one field emitted by the EM transmitter demonstrates the detection of probes and other nearby precalibrated instruments (C).

style) was placed, and K-wires were inserted into the pedicle. Finally, custom-attached DS Taps and Expedium style (Viper) cannulated lumbar pedicle screws were placed using custom DS NAV screwdrivers (DePuy Spine, a Johnson \& Johnson company, Raynham, Massachusetts). 
Intracadaveric analysis was conducted to compare EM guidance to conventional fluoroscopy by a radiologist blinded to the protocol (J.A.C.). Intraoperative variables for analysis included total fluoroscopic time and mean fluoroscopic time per screw. Fluoroscopy time was taken from a timer on the mobile C-arm. For the EM group, fluoro time required for initial registration was divided by the number of screws placed; in total 3 anteriorposterior and 3 lateral static fluoroscopic shots are required to register the entire lumbosacral spine.

Each specimen underwent a postoperative CT with reconstructions; radiographic analysis was conducted by an independent, blinded radiologist (J.A.C.). Each screw placement was rated for pedicle breach, defined as penetration through the cortical edge of the pedicle, while vertebral breach was defined as penetration of the vertebral wall. Critical breach was defined as a cortical breach that encroached upon neurovascular elements (perforation through medial/inferior aspect of pedicle or anterior cortex of vertebral body). The ideal trajectory was defined as screw placement precision toward medial ventral aspect of the vertebral body such that bilateral screws would converge while remaining entirely within the pedicles. The trajectory was rated accordingly: 0 (ideal), 1 (1-3 $\mathrm{mm}$ off ideal), 2 ( $>3 \mathrm{~mm}$ but $<5 \mathrm{~mm}$ off ideal), 3 ( $\geq 5 \mathrm{~mm}$ off ideal). In comparing trajectories, overall number of pedicle breaches, vertebral breaches, and critical breaches were evaluated for EM guidance compared to conventional fluoroscopy. In addition, lumbar spine segment (L1-L5) breaches were evaluated in a separate comparison to specifically study the breach rates for lumbar pedicle screw placement.

Statistical analysis was conducted utilizing Wilcoxon MatchedPairs Signed-Ranks Tests for total fluoroscopy time, mean fluoroscopy time/screw, and trajectory. Fisher's exact tests were used to compare rates of pedicle breach, vertebral breach, and critical breach. A $P$-value $<.05$ was considered significant.

\section{RESULTS}

Five cadaveric specimens underwent bilateral screw placement from L1 to S1, using conventional fluoroscopy (CF) on one side and EM guidance (EM) on the matching contralateral side. Data for total fluoroscopy time was excluded for one specimen (Spine 5); L1 was not instrumented in Spine 5 due to previous kyphoplasty, and S1 was not instrumented due to an abnormal anatomic relationship with the pelvis that confounded screw placement. As such, a total fluoroscopy time for that specimen would be confounded by the exclusion of two levels. Overall, the average total fluoroscopy time/specimen was $383.3 \pm 255.6$ seconds for $\mathrm{CF}$ and $160.5 \pm 79.6$ seconds for EM (ns). The overall mean fluoroscopy time per screw was $58.9 \pm 44.7$ seconds for $\mathrm{CF}$ compared to $27.4 \pm 13.5$ seconds for $\operatorname{EM}(P=.0003)$.

Trajectory and breach were analyzed on postoperative CT scans. Mean trajectory rating was $1.1 \pm 1.1$ for $\mathrm{CF}$ and $1.4 \pm 0.2$ for $\mathrm{EM}$ (ns). The number and distribution of breaches are demonstrated in Figure 2, and the overall breach rates are shown in Table 1.
Overall, the EM screws demonstrated 3 more pedicle breaches but 3 fewer critical breaches than the CF screws (ns). Figure 3 summarizes the results for lumbar pedicle screw placements

\section{Figure 2. Distribution of All Breaches}

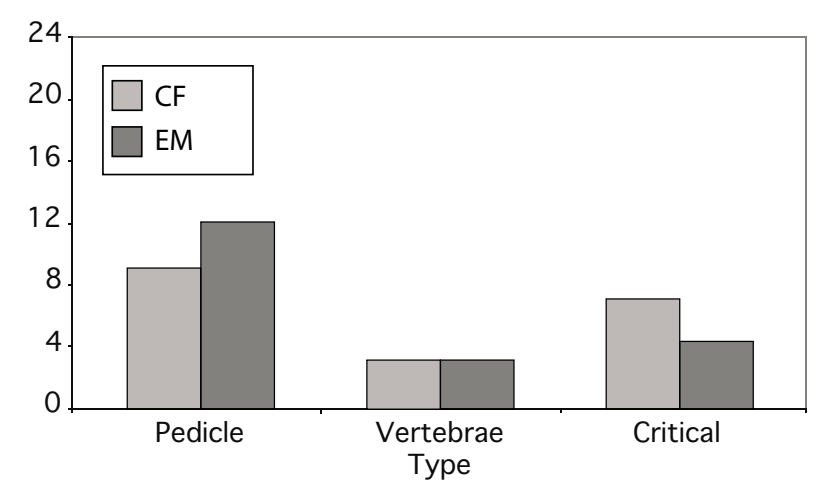

The overall number of breaches was compared for conventional versus EM-guided navigation for each type of breach. While the data showed a trend toward a higher critical breach rate for conventional fluoroscopy, analysis did not reach statistical significance.

Table 1. Rates of Breach by Type for Conventional Fluoroscopy (CF) Versus Electromagnetic Guidance (EM)

\begin{tabular}{rcccc}
\hline \multicolumn{4}{c}{ Overall Breach Rate (\%) } & \multicolumn{2}{c}{ Lumbar Breach Rate (\%) } \\
\hline Breach Type & CF & EM & CF & EM \\
Pedicle & 32.1 & 42.8 & 33.3 & 33.3 \\
Vertebrae & 10.7 & 10.7 & 8.3 & 12.5 \\
Critical & 25.0 & 14.2 & 25.0 & 0.0 \\
\hline
\end{tabular}

Figure 3. Distribution of Breaches in the Lumbar Spine

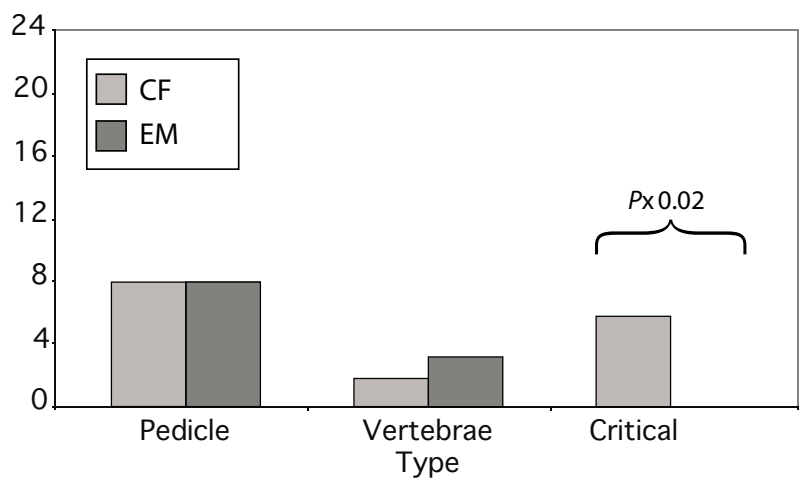

The number of breaches in the lumbar spine (L1-L5) was compared for conventional versus EM-guided navigation for each type of breach. There was a significantly higher number of critical breaches for conventional fluoroscopy in the lumbar spine.

(L1-L5), excluding the sacral screws. When lumbar screws were evaluated alone, EM-guided screws demonstrated 1 more vertebral breach but 6 fewer critical breaches. In comparing critical breaches in the lumbar spine, there was a significant difference between EM guidance and $\mathrm{CF}$ guidance $(P=.02)$. 
Recent advances in imaging technology have facilitated percutaneous placement of pedicle screws. Minimally invasive spine surgery is integrally tied to such advances, as imaging tools must act as the "eyes" of the surgeon when placing instrumentation. Together with other minimally invasive spine surgery technology, these advances have enabled key alterations in surgical technique and approach that reduce open exposure of anatomy and incision length. Technical comparisons of these techniques to more traditional methods rest on demonstrating at least equipoise in operative time, radiation exposure, and precision of instrumentation. While not the only imaging technology available for minimally invasive spine surgery, electromagnetic imaging has unique advantages. Unlike optical imaging tracking systems, EM does not require line-of-sight between the reference device, the tracking apparatus, and the calibrated stereotactic probe or instrument. Without the need for optical cameras, the EM system additionally carries a smaller footprint in the OR, occupying a smaller amount of space in an already small working space.

In addition, optical navigation involves both active and passive tracking. Passive tracking is wireless on the field but requires larger cumbersome attachments; with these attachments, the tools need to be tracked by a camera in the background reflecting the infrared off the optical spheres. The active optical tracking instruments are also wired to generate pulses back to the remote camera, though some have battery packs attached to them. In comparison, the screwdriver is an integrated EM tool that interchanges with the custom minimal-access spine instruments; the wire is well managed and secured in the sterile field similar to the fiberoptic light bundles used in endoscopy.

While certain interference has been reported from metal objects within the OR, our cadaveric procedures, designed to simulate similar live operations, did not encounter interference-related problems. In particular, the EM instruments were made of low-ferrous or non-ferrous material that compensated or eliminated distortion that would have been associated with the EM field. Thus, with such potential advantages, our study aimed to compare novel electromagnetic guidance technology to conventional fluoroscopy for the placement of percutaneous pedicle screws in the lumbosacral spine.

Our study utilized a 2-dimensional real-time imaging guidance tool to percutaneously place pedicle screws. While such analyses are important as first steps for integrating navigational technology in minimally invasive spine surgery, a 3-dimensional guidance tool might be more applicable. This is especially true for patients with complex anatomic deformities such as coincidental scoliosis, and for patients undergoing "redo" minimally invasive procedures. Though one could consider this a study limitation, it reflects more of a limitation in current technology. In demonstrating the applicability of a 2-dimensional image guidance method to minimally invasive lumbosacral pedicle screw placement, our data support the future integration and investigation of 3-dimensional rendering technology.

Though limited to 5 cadavers, our study demonstrated significant reduction in fluoroscopy time spent per level from L1 to S1, and a reduction in critical breaches in the lumbar spine (L1 L5). The inability to show a statistically significant difference in breach rates when all lumbar and sacral screws were considered may be an indication of the relative precision of this technology in placing lumbar versus sacral pedicle screws. However, the small sample size of this study precludes us from drawing such a conclusion, as we cannot rule out beta error in the comparison of all screws (lumbar and sacral).

At a minimum, these results demonstrate the non-inferiority of EM guidance to conventional fluoroscopy. They may also indicate that EM guidance could provide some benefit in the placement of percutaneous pedicle screws in the lumbar spine. Though an ideal analysis would compare patients in a randomized fashion, our cadaveric study provides a strong foundation for such future studies. In spinal fusions, reduction of operative time remains an important goal; reduction in operative time may reduce blood loss, anesthesia time, and complication rates. Our results demonstrated an average reduction of $53.5 \%$ in fluoro time per screw, which, for long-segment spine fusions could translate into a significant reduction in overall fluoroscopy and operative time. These results in the lumbar spine mirror comparable results from other studies in the thoracic and lumbar spine for non-percutaneously placed screws. ${ }^{5,6}$ For example, in a study of lumbar pedicle screws placed via open approach using conventional fluoroscopy, Sagi et al. found a critical perforation rate of $22 \%$ (compared to our rate of $25.0 \%) .{ }^{6}$ However, while Sagi et al. found a critical perforation rate of $5 \%$ for lumbar screws placed via EM guidance, our results showed no critical perforations for EM-guided screws. ${ }^{6}$ This may reflect several differences in study methods. First, Sagi et al. used an open approach to visualize the anatomy and insert the screws, compared to our percutaneous technique. While a direct comparison of our results and theirs would not be statistically sound, it is encouraging that our results are at least at equipoise with those from an open posterior exposure. Second, Sagi utilized postoperative direct dissection to evaluate breach and critical perforation.

Although the sensitivity and specificity of dissection versus CT for detecting such results could be debated, it is clear that postoperative CT is more applicable clinically as it would be the most likely method of assessment in postoperative patients. Regardless, both our study and those by Sagi demonstrate a clear role for electromagnetic guidance tools in thoracolumbar pedicle screw placement. In addition, our study demonstrates the applicability of this technique in minimally invasive spine surgery as a method for guiding percutaneously placed screws.

Minimally invasive procedures such as percutaneous pedicle screw fixation have been shown to be safe and reliable, but they 
demand an effective imaging tool to accurately predict target trajectory for screw placement. ${ }^{7}$ Other recent studies have evaluated pedicle screw placement from a minimally invasive perspective. Early versions of real-time tracking using spine imaging included optical tracking systems which demonstrated a thoracic pedicle cortex breach rate of $19.2 \%$, compared to the thoracic pedicle breach rate of $8 \%$ published in cadaveric studies of electromagnetic field tracking in the thoracic spine. ${ }^{3,5}$ Similarly, published experience with real-time tracking for percutaneous pedicle screw placement in the lumbar spine has been encouraging. In a study of patients with previous fusion undergoing new instrumentation, Lim et al. found only $4.1 \%$ of 122 pedicle screws placed in the lumbar spine to have unintended cortical violations on follow-up after using frameless stereotaxy for guidance. ${ }^{8}$ Our pedicle breach rates for conventional fluoroscopy and EM guidance were at equipoise, but there were significantly fewer critical breaches among EMguided screws in the lumbar spine. Our inability to demonstrate superiority in breach rates of one method over another was likely secondary to a small sample size. However, while further studies are necessary, EM guidance may be an important tool to make percutaneous screw placement in the lumbar spine, not only more efficient, but also more precise.

Our study demonstrates important findings regarding the use of EM guidance in lumbar fusion techniques. However, it represents a limited examination in a cadaveric model. The small number of screws placed represents one limitation, though we were able to show a difference in time/screw and critical breaches even with a small sample. Thus, our study provides a foundation upon which to base a clinical examination of this subject. Despite such a limitation, our study demonstrates a role for electromagnetic guidance in minimally invasive spinal surgery.

\section{CONCLUSIONS}

Minimally invasive spine fusion in the lumbosacral region requires an accurate real-time imaging modality for screw trajectory guidance. Electromagnetic navigational systems provide safe and effective tools for intraoperative guidance. Our cadaveric study suggests that EM guidance may be more efficient than conventional fluoroscopic technique. Further clinical studies are needed to further elucidate differences in these techniques; this study provides a foundation and justification for such research. In particular, the introduction of 3-dimensional navigation with EM guidance could improve accuracy and efficiency in percutaneous pedicle screw placement.

Justin F. Fraser, MD, Ron Von Jako, MD, John A. Carrino, MD, MPH, and Roger Härtl, MD

From Department of Neurological Surgery, Weill Medical College of Cornell University, New York Presbyterian Hospital, New York, NY (Fraser and Härtl); Surgical Development Leader, GE Healthcare Surgery (Von Jako); and Division of Musculoskeletal Radiology, Russell H. Morgan Department of Radiology and Radiological Science, Johns Hopkins University School of Medicine, Baltimore, MD (Carrino)

Ron Von Jako, MD, is an employee and shareholder in GE Healthcare Surgery. Cadaveric specimens were provided by GE Healthcare Surgery. Drs. Fraser, Carrino, and Härtl are not paid employees and do not receive personal stipends from GE Healthcare Surgery.

Address correspondence to Roger Härtl, MD, Department of Neurological Surgery, Weill Medical College of Cornell University, New York Presbyterian Hospital, 525 E. 68th St., Box 99, New York, NY 10021, (email: roger@hartlmd.net)

This manuscript was submitted June 17, 2007, and accepted for publication November 20, 2007.

\section{REFERENCES}

1. Wiesner L, Kothe R, Ruther W. Anatomic evaluation of two different techniques for the percutaneous insertion of pedicle screws in the lumbar spine. Spine. 1999;24(15):1599-1603.

2. Laine T, Lund T, Ylikoski M, Lohikoski J, Schlenzka D. Accuracy of pedicle screw insertion with and without computer assistance: a randomised controlled clinical study in 100 consecutive patients. Eur Spine J. 2000;9(3):235-240.

3. Kim KD, Patrick Johnson J, Bloch BO, Masciopinto JE. Computerassisted thoracic pedicle screw placement: an in vitro feasibility study. Spine. 2001;26(4):360-364.

4. Bolger C, Wigfield C. Image-guided surgery: applications to the cervical and thoracic spine and a review of the first 120 procedures. J Neurosurg. 2000;92(2 Suppl):175-180.

5. Sagi HC, Manos R, Park SC, Von Jako R, Ordway NR, Connolly PJ. Electromagnetic field-based image-guided spine surgery part two: results of a cadaveric study evaluating thoracic pedicle screw placement. Spine. 2003;28(17):E351-354.

6. Sagi HC, Manos R, Benz R, Ordway NR, Connolly PJ. Electromagnetic field-based image-guided spine surgery part one: results of a cadaveric study evaluating lumbar pedicle screw placement. Spine. 2003;28(17):20132018.

7. Wiesner L, Kothe R, Schulitz KP, Ruther W. Clinical evaluation and computed tomography scan analysis of screw tracts after percutaneous insertion of pedicle screws in the lumbar spine. Spine. 2000;25(5):615621.

8. Lim MR, Girardi FP, Yoon SC, Huang RC, Cammisa FP, Jr. Accuracy of computerized frameless stereotactic image-guided pedicle screw placement into previously fused lumbar spines. Spine. 2005;30(15):1793-1798. 